\title{
Disease Severity Adversely Affects Delivery of Dialysis in Acute Renal Failure
}

\author{
Helmut Schiffl \\ Department of Internal Medicine, University of Munich, Munich, Germany
}

\section{Key Words}

Acute renal failure $\cdot$ Intermittent hemodialysis $\cdot$ Dialysis dose

\begin{abstract}
Background/Aims: Methods of intermittent hemodialysis (IHD) dose quantification in acute renal failure (ARF) are not well defined. This observational study was designed to evaluate the impact of disease activity on delivered single pool $\mathrm{Kt} / \mathrm{V}_{\text {urea }}$ in ARF patients. Methods: 100 patients with severe ARF (acute intrinsic renal disease in 18 patients, nephrotoxic acute tubular necrosis in 38 patients, and septic ARF in 44 patients) were analyzed during four consecutive sessions of IHD, performed for 3.5-5 h every other day or daily. Target IHD dose was a single pool Kt/ $\mathrm{V}_{\text {urea }}$ of 1.2 or more per dialysis session for all patients. Prescribed $\mathrm{Kt} / \mathrm{V}_{\text {urea }}$ was calculated from desired dialyzer clearance $(\mathrm{K})$, desired treatment time (t) and anthropometric estimates for urea distribution volume (V). The desired clearance (K) was estimated from prescribed blood flow rate and manufacturer's charts of in vivo data obtained in maintenance dialysis patients. Delivered single pool $\mathrm{Kt} / \mathrm{V}_{\text {urea }}$ was calculated using the Daugirdas equation. Results: None of the patients had prescription failure of the target dose. The delivered IHD doses were substantially lower than the prescribed Kt/V values, particularly in ARF patients with sepsis/septic shock. Stratification according to disease severity revealed that all patients with isolated ARF, but none with 3 or more organ failures and none
\end{abstract}

who needed vasopressive support received the target dose. Conclusion: Prescription of target IHD dose by single pool $\mathrm{Kt} / \mathrm{V}_{\text {urea }}$ resulted in suboptimal dialysis dose delivery in critically ill patients. Numerous patient-related and treatmentimmanent factors acting in concert reduced the delivered dose.

Copyright $\odot 2007$ S. Karger AG, Basel

\section{Introduction}

The increasing number of multi-morbid patients admitted to medical or surgical services has been accompanied by a greater incidence of acute renal dysfunction in hospitalized patients. Despite its potential reversibility, the development of hospital-acquired acute renal failure (ARF) represents a major barrier for the recovery of the patient. Even small changes in renal function are associated with substantial increases in mortality and the outcome of critically ill patients with ARF requiring acute renal replacement therapy has remained distressingly poor over the decades [1].

It is not yet clear which strategies of renal replacement therapy provide the optimal choice to improve patient morbidity and mortality in ARF. Several studies have shown that more intensive provision of dialysis may improve the survival of intensive care unit (ICU) patients with ARF [2-4]. While both the minimally adequate as well as the optimal doses are unknown, it is clear that the

\section{KARGER}

Fax +4161306 1234 E-Mail karger@karger.ch www.karger.com

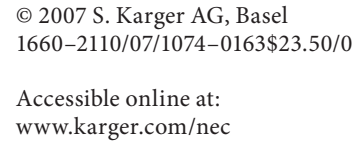


dose provided as estimated by urea kinetic modeling is often markedly lower than the prescribed dose [5-11]. Evanson et al. [6] found that although $49 \%$ of $40 \mathrm{ARF}$ patients were prescribed a Kt/V of $<1.2$, the $\mathrm{Kt} / \mathrm{V}$ delivered was below this level in the majority of patients (70\%). These data indicate that dialysis delivery is mostly suboptimal in ARF.

There is no doubt that an inadequately prescribed dialysis dose results in delivery failure. Further reasons for the discrepancy between prescribed and delivered dialysis in the setting of ARF are treatment-related factors (catheter dysfunction, recirculation, decreased dialyzer function) $[6-8,11]$. Whether or not demographic factors affect delivery of dialysis dose in the setting of ARF is not clear.

This prospective cohort study examined the utility of single pool Kt $/ \mathrm{V}_{\text {urea }}\left(\mathrm{spKt} / \mathrm{V}_{\text {urea }}\right.$ ) for prescription of a target dose in 3 groups of patients with different causes of ARF. Severity of disease was identified as a factor that impeded effective delivery of prescribed dialysis in ARF patients.

\section{Patients and Methods}

\section{Study Design}

Patients with severe ARF requiring intermittent hemodialysis (IHD) were eligible for enrolment in this prospective observational study during a period from 1997 to 2005. Patients were treated at the Department of Internal Medicine (Medizinische Klinik-Innenstadt) of the University of Munich or KfH Renal Centers in Munich-Laim or in Bad Reichenhall. Measurements of $\mathrm{spKt} / \mathrm{V}$ were part of routine practice of renal replacement therapy for ARF in these centers. The investigator had no influence on the general medical care of the patients. No rigid alternate day or daily dialysis schedule was employed and treatment decisions were made on a day-to-day basis according to the individual patient's needs. The study protocol was approved by the Institutional Review Board of each participating site. Informed consent was obtained from the patients or from their next of kin.

\section{Study Population}

Patients were included in the study if they had ARF secondary to acute intrinsic renal disease, clinically diagnosed toxic acute tubular necrosis (ATN) or sepsis based on the criteria set by the American College of Chest Physicians [12]. Exclusion criteria were participation in another clinical trial, pregnancy, organ transplantation, preexisting chronic renal insufficiency (based on history, abnormal ultrasound or urinalysis, elevated serum creatinine and/or a reduction in the calculated glomerular filtration rate prior to the acute disease), and isolated ultrafiltration for hypervolemia. Patients were also excluded when they had bleeding disorders contradicting systemic heparin anticoagulation (active gastrointestinal bleeding, recent surgery, thrombocytopenia or consumption coagulopathy) and concomitant therapy with plasmapheresis. Patients who had, or were at risk of, cerebral edema received continuous renal replacement therapies.

\section{Intermittent Hemodialysis}

Each patient had 2 polyurethane dialysis catheters placed percutaneously in two central veins, either femoral veins (length 25 $\mathrm{cm}$ ) or the internal jugular or the subclavian vein (length 15 or 20 $\mathrm{cm})$. Dialysis treatments were performed with machines that had volumetric ultrafiltration control (Fresenius MTS 2008 C, Fresenius, Homburg v.d. Höhe, Germany, or Gambro AK 200, Gambro, Hechingen, Germany). Single-use polysulfone dialyzers (F 60 Fresenius, Germany) and bicarbonate dialysate were used exclusively. Blood flow rates ranged between 250 and $350 \mathrm{ml} / \mathrm{min}$; the dialysate flow rate was set at $500 \mathrm{ml} / \mathrm{min}$. The dialysis fluid met the microbiological standards of the European Pharmacopoeia of $<100 \mathrm{CFU} / \mathrm{ml}$ and $<0.25$ endotoxin units/ml. IHD was performed according to the patient's clinical status, the frequency ranged from alternate day to daily sessions.

\section{Clinical Data Acquisition}

Baseline demographic characteristics were prospectively collected from the patient's hospital records. These included age, gender, body weight and height at admission. At commencement of IHD the cause of ARF (based on history, clinical investigation, renal ultrasound, urinalyses, blood parameters and/or kidney biopsy) was acute renal disease (glomerulonephritis, vasculitis, tubulointerstitial nephritis, atheroembolism) in 18 patients, nephrotoxic ATN (antibiotics, cytostatic drugs, contrast media, hemoglobinuria and myoglobinuric disorders) in 38 patients, and sepsis/septic shock in 44 patients. The severity of illness in each patient was determined using the Acute Physiological and Chronic Health Evaluation (Apache II) score before the first dialysis session. The number of organ failures was calculated using the multiple systems organ failure score [13]. ARF-related biochemical parameters such as blood urea nitrogen (BUN), serum creatinine levels, and urine volume were also recorded.

Dialysis treatment data included the site of central venous catheter, intra-dialysis blood flow rates (documented at 30-min intervals), pre- and post-dialysis weight (measured by stand-on or chair scales in mobile patients and by bed scales in bedridden patients), duration of dialysis sessions, pre- and post-dialysis BUN, ultrafiltration rate, anticoagulation, post-dialysis judgment of dialyzer patency, mean arterial pressure prior to the hemodialysis session and need for pressor support (except renal dose of dopamine). Blood samples were again drawn in 10 patients of each group 60 min after hemodialysis.

Prescribed Dose of IHD

Before each treatment, a prescribed Kt/V was determined according to the dialyzer manufacturer's specifications, the in vivo clearance of urea $(\mathrm{ml} / \mathrm{min})$ was determined in maintenance dialysis patients, and the dialysis prescription time and the anthropometric estimates of total body water (the urea volume distribution) according to the equation of Watson et al. [14]. The desired clearance was estimated from prescribed blood flow and the manufacturer's charts of in vivo data. Patients in each etiologic ARF group were dialyzed with a projected $\mathrm{spKt} / \mathrm{V}$ of 1.2 per session, which was derived from contemporary clinical practice guidelines which recommended a minimum delivered spKt/V of 1.2 for patients with ESRD $[15,16]$. 
Delivered Dose of IHD

The pre-dialysis BUN sample was drawn from the femoral catheter immediately before dialysis. The post-dialysis BUN sample was obtained using the stop flow method (blood flow rate $50-100 \mathrm{ml} / \mathrm{min}$ for $20 \mathrm{~s}$ ). The delivered dialysis dose was assessed by spKt/V using the second-generation equation of Daugirdas [17]:

$$
\operatorname{spKt} / \mathrm{V}=-\ln (\mathrm{R}-0.008 \mathrm{t})+(4-3.5 \mathrm{R}) \mathrm{UF} / \mathrm{W}
$$

where $\mathrm{R}$ is the post-BUN/pre-BUN dialysis ratio, $\mathrm{t}$ is the delivered dialysis time in hours, UF is the volume of fluid removal during dialysis in liters and $\mathrm{W}$ is the post-dialysis weight in kilograms.

\section{Calculation of Post-Dialysis Urea Rebound}

Post-dialysis urea rebound (PDUR) was calculated as the percentage of the increase in serum urea levels at $60 \mathrm{~min}$ compared with urea levels immediately post-dialysis:

$$
\begin{aligned}
\text { PDUR }= & \text { (urea after } 60 \mathrm{~min}-\text { urea post-dialysis }) \\
& \times 100 / \text { urea post-dialysis. }
\end{aligned}
$$

\section{Statistics}

Data are presented as mean \pm SD or as percentage. For the demographic variables, an analysis of variance was used to evaluate the continuous values, whereas Fisher's exact test was used to evaluate the categorical variables. The Generalized Estimating Equation method statistical procedure for longitudinal data analysis with multiple observable vectors for the same subject was also used in the data analysis. This procedure is a repeated measures analysis for corrected dichotomous outcomes and a set of predictors [18]. All tests of significance were two-sided and differences were considered significant for $p$ values of $<0.05$. SAS version 6.12 (SAS Institute, Inc., Cary, N.C., USA) was used for all analyses.

\section{Results}

\section{Characteristics of the Study Cohort}

One hundred patients with ARF requiring IHD were selected for this observational study according to disease severity. Eighty $(80 \%)$ of the patients were treated in the ICU, and of those, 50 patients were on ventilator therapy and 55 patients received vasopressors. Compared to body weight at admission patients with septic shock had a clinically relevant and statistically significantly higher weight at the start of IHD (mean weight gain $8 \mathrm{~kg}$ ).

The patients with acute renal disease had isolated ARF, a lower severity of acute illness compared to other patients, a better survival (as judged by the in-hospital mortality), but a significantly higher proportion of these patients needed dialysis at discharge. Septic patients were critically ill (59\% with septic shock), had ARF as part of multiple organ failure syndrome with an excessive inhospital case mortality rate (table 1 ).

Single Pool Kt/V $\mathrm{V}_{\text {urea }}$ in Acute Renal Failure

\begin{tabular}{|c|c|c|c|}
\hline & $\begin{array}{l}\text { Acute renal } \\
\text { disease }\end{array}$ & $\begin{array}{l}\text { Toxic } \\
\text { ATN }\end{array}$ & Sepsis \\
\hline Number of patients & 18 & 38 & 44 \\
\hline \multicolumn{4}{|l|}{ At admission } \\
\hline Age, years & $47 \pm 8$ & $58 \pm 8$ & $60 \pm 13$ \\
\hline Gender, m/f & $12 / 6$ & $23 / 15$ & $24 / 20$ \\
\hline Body weight, kg & $74 \pm 15$ & $71 \pm 13$ & $65 \pm 10$ \\
\hline \multicolumn{4}{|l|}{ At start of IHD } \\
\hline BUN, mg/dl & $76 \pm 10$ & $72 \pm 16$ & $80 \pm 12$ \\
\hline Creatinine, mg/dl & $4.8 \pm 1.1$ & $4.5 \pm 1.4$ & $4.3 \pm 1.5$ \\
\hline Oliguria, $\%$ & 44 & 46 & 48 \\
\hline MAP, mm Hg & $110 \pm 10$ & $105 \pm 13$ & $78 \pm 8^{* *}$ \\
\hline APACHE II & $10 \pm 2^{*}$ & $19 \pm 3^{* *}$ & $32 \pm 3$ \\
\hline Organs failing & $1 \pm 0^{*}$ & $1.6 \pm 0.2^{* *}$ & $3.1 \pm 0.3$ \\
\hline Body weight, kg & $75 \pm 16$ & $73 \pm 12$ & $73 \pm 11$ \\
\hline \multicolumn{4}{|l|}{ Outcome parameters } \\
\hline Hospital case fatality rate, $\%$ & $6^{*}$ & $21^{* *}$ & 70 \\
\hline \multicolumn{4}{|l|}{ Need for dialysis } \\
\hline after discharge, $\%$ & $50^{*}$ & 3 & 0 \\
\hline
\end{tabular}

Table 1. Patient characteristics at admission, at start of dialysis and at discharge

Unless otherwise indicated, the data are mean \pm SD.

${ }^{*} \mathrm{p}<0.05$ vs. nephrotoxic ATN and sepsis or vs. nephrotoxic ATN and acute renal disease; ${ }^{* *} \mathrm{p}<0.05$ vs. acute renal disease or sepsis.

Table 2. Prescribed and delivered spKt/V in the three ARF patient groups (mean $\pm \mathrm{SD})$

\begin{tabular}{llll}
\hline & $\begin{array}{l}\text { Acute renal } \\
\text { disease }\end{array}$ & ATN & Septic ARF \\
\hline $\begin{array}{llll}\text { Blood flow rate, } \mathrm{ml} / \mathrm{min} \\
\quad\end{array}$ & & \\
$\quad \begin{array}{l}\text { Prescribed } \\
\text { Delivered }\end{array}$ & $271 \pm 37$ & $269 \pm 34$ & $269 \pm 40$ \\
$\begin{array}{l}\text { Duration of session, min } \\
\quad \text { Prescribed }\end{array}$ & $237 \pm 31$ & $215 \pm 30$ & $217 \pm 27$ \\
$\quad$ Delivered & $231 \pm 29$ & $219 \pm 28$ & $220 \pm 25$ \\
spKt/V & & & \\
$\quad$ Prescribed & $1.36 \pm 0.17$ & $1.28 \pm 0.06$ & $1.28 \pm 0.07$ \\
$\quad$ Delivered & $1.31 \pm 0.10$ & $1.15 \pm 0.12$ & $0.89 \pm 0.04^{*}$ \\
PDUR, \% & $15 \pm 2$ & $17 \pm 1$ & $22 \pm 2$ \\
\hline
\end{tabular}

PDUR = Post-dialysis urea rebound.

${ }^{*} \mathrm{p}<0.05$ vs. acute renal disease or toxic ATN.

Characteristics of Hemodialysis Sessions

The prescription of IHD was similar in all groups of ARF patients; each patient had a prescribed target dose of a spKt/V of 1.2 or more per session (table 2). All patients 
Table 3. Delivery of target IHD dose in three ARF patient groups

\begin{tabular}{lcr}
\hline & \multicolumn{2}{l}{$\mathrm{Kt} / \mathrm{V}_{\text {urea }}$} \\
\cline { 2 - 3 } & $<1.2$ & $\geq 1.2$ \\
\hline Acute intrinsic renal disease & 0 of 18 & 18 of 18 \\
Toxic acute tubular necrosis & 11 of 38 & 27 of 38 \\
Septic ARF & 44 of $44^{*}$ & 0 of 44 \\
\hline
\end{tabular}

Values are the number of patients.

${ }^{*} \mathrm{p}<0.05$ vs. acute renal disease or toxic acute tubular necrosis.

Table 4. IHD dose delivered in three groups of ARF patients stratified according to disease severity

\begin{tabular}{lrr}
\hline & \multicolumn{2}{l}{$\mathrm{Kt} / \mathrm{V}_{\text {urea }}$} \\
\cline { 2 - 3 } & $<1.2$ & $\geq 1.2$ \\
\hline $\begin{array}{l}\text { Number of organs failing } \\
1\end{array}$ & 0 of 35 & 35 of 35 \\
2 & 10 of 20 & 10 of 20 \\
3 or more & 45 of $45^{*}$ & 0 of 45 \\
$\begin{array}{l}\text { Pressor support } \\
\text { No vasoactive drugs }\end{array}$ & 0 of 45 & 45 of 45 \\
$\quad$ Vasopressor & 55 of 55 & 0 of 55 \\
\hline
\end{tabular}

Values are the number of patients.

${ }^{*} \mathrm{p}<0.05$ vs. acute renal disease or toxic ATN.

had two catheters in central veins, they all received heparin and there was no clotted dialyzer. Given the low predialysis blood pressure and the need for vasopressors, hypotensive episodes necessitating intervention (administration of saline, reduction of ultrafiltration rate) were low, even in septic patients (5\% acute renal disease vs. $7 \%$ ATN vs. $10 \%$ septic ARF, respectively). No dialysis session was prematurely terminated. The three patient groups differed in the frequency of IHD per day ( 0.5 per day for acute renal disease, 0.7 per day for ATN, and 1.0 per day for septic ARF). There were no statistically significant differences in ultrafiltration rate per session (mean ultrafiltration rate was $1,765 \mathrm{ml} / \mathrm{session}$ for acute renal disease, $1,665 \mathrm{ml} /$ session for the patient group with nephrotoxic ATN, and 1,825 ml/session for septic patients.

\section{Delivered IHD according to Disease Severity}

There was no statistically significant difference between prescribed and delivered $\mathrm{Kt} / \mathrm{V}$ in patients with acute renal disease. The delivered dialysis dose was significantly lower than the prescribed KT/V in patients with ATN or septic ARF. Delivery failure was more pronounced in patients with septic ARF (tables 2,3). The delivery of IHD dose was loosely correlated to the number of failing organs (table 4) and none of the patients on vasopressor support reached the target dose of a spKt/ $\mathrm{V}_{\text {urea }}$ of 1.2 (table 4).

Post-dialysis rebound tended to be higher in septic patients (table 2).

\section{Discussion}

There are two types of factors that give rise to underdelivery of prescribed IHD dose as measured by spKt/ $\mathrm{V}_{\text {urea }}$ in the setting of ARF: those pertaining to the patient and those related to the therapy itself. The data of this observational study highlight the complex interactions between the patient's severity of acute illness and the delivery of the IHD dose. The greater inability to deliver the prescribed dose to critically ill patients with severe ARF is a new finding. The discrepancy between the present investigation and previous analyses may be well explained by substantial differences in the number of participants, the case mix of causes of ARF and the inclusion or exclusion of patients with prescription failure $[6,8]$.

$\mathrm{SpKt} / \mathrm{V}_{\text {urea }}$ is a generally accepted method to measure the delivered dose of dialysis in maintenance dialysis patients. However, the reliability and utility of the bloodbased simplified IHD quantification formula of urea kinetic modeling may be not the same in the setting of ARF as in ESRD as the fundamental assumptions of measurement of spKt $/ \mathrm{V}_{\text {urea }}$ may be violated, at least in critically ill patients with ARF necessitating IHD. Measurements of $\mathrm{spKt} / \mathrm{V}_{\text {urea }}$ assumes steady-state urea nitrogen appearance, constant dialyzer clearance at a given blood and dialysate flow rate, and a 'well-mixed' urea volume of distribution, identical to the total body water, with no compartmentalization of urea distribution. Due to the nature of an acute life-threatening illness; however, patients with severe ARF are rarely in a steady-state.

Whereas delivered $\mathrm{Kt} / \mathrm{V}_{\text {urea }}$ values in patients with isolated ARF were slightly lower than the prescribed Kt/ $\mathrm{V}_{\text {urea }}$ values in analogy to maintenance dialysis patients, a substantial difference (by 30\%) between the prescribed and delivered dialysis dose was found in critically ill pa- 
tients with ARF. The discrepancy between the prescribed and delivered dose of IHD observed in the present study was not caused by the failure to prescribe a Kt/ $\mathrm{V}_{\text {urea }}$ of $>1.2$.

There is compelling evidence that volume expansion, typically seen in ICU patients who suffer from shock and multi-organ insults, represents a major factor contributing to the under-delivery of the prescribed IHD dose. This expansion of body water compartments is the consequence of therapeutic volume resuscitation in the setting of increased capillary permeability often seen in patients with sepsis/septic shock. The expansion is usually not subtle and may sometimes exceed 30-40 liters in a given patient [19]. This distortion of body habitus obviously renders invalid the calculation of body water from anthropometric measurements derived from measurements in normal healthy adults. Himmelfarb et al. [20] showed in critically ill patients with ARF that determinations of total body water by anthropometric measurements (Watson, Hume-Weyer, Chertow formulae) yielded significantly lower measures compared to total body water values determined by physiological formulae or bioelectrical impedance analysis. Moreover, V determined by formal urea kinetic modeling or by isotope methods was reported to average 65 or $64.3 \%$ of body weight in critically ARF patients [21,22]. In support of the over-hydration factor, Kanagasundaram et al. [10] found that the ratio of modeled to anthropometric urea volume of distribution was highest at the point of initiating dialysis, and this diminished over time as regular dialysis therapy was applied. Thus, attempts to use formulas such as Watson's (used in this study) for the prescription of dialysis will greatly overestimate the delivered dose.

Secondly, critically ill ARF patients have high protein catabolic rates $(>1.5 \mathrm{~g} / \mathrm{kg} /$ day) and substantial nitrogen deficits (6 g/day). This catabolic state, which can vary from hour to hour, may affect the actual delivered dose of dialysis by resulting in accelerated intra-dialytic urea generation that needs to be taken into account when measuring blood-based methods of IHD dosing. However, Evanson et al. [7] demonstrated that the patient's catabolic rates, as estimated by the urea nitrogen appearance, had no significant effect on the delivered Kt/V. Other potential problems with delivery of dialysis dose include post-hemodialysis BUN rebound and cardiopulmonary recirculation which may influence the post-treatment BUN levels. These factors probably had little influence on the results of this study because firstly, all angio-accesses used were venovenous and therefore there was no cardio- pulmonary recirculation. Secondly, the stop-flow method with timed blood sample collection was developed to diminish the effects of post-dialysis BUN rebound. There was no significant difference between the time of collection (20 s) among the three etiologic groups of ARF.

The second potential stumbling block with blood-derived clearance-based methods relates to IHD treatmentrelated factors: the nature of the angio-access and the need for anticoagulation. Access recirculation and poor blood flows with inadequate catheter function are inherent limitations of acute IHD and have a negative impact on the $\mathrm{spKt} / \mathrm{V}_{\text {urea }}$ delivered. Numerous clinical studies have demonstrated that the frequency of these drawbacks of temporary venous catheters depends on the site of insertion, technique of insertion (tunneled vs. conventional) and the length of catheter used. Liangos et al. [9] described a relation between delivered dialysis dose and the use of two-lumen femoral catheters. These authors found a high blood flow rate and a decrease in urea reduction ratio with femoral compared to non-femoral catheters. Klouche et al. [11] showed that tunneled two-lumen femoral catheters were associated with higher delivered spKt/ $\mathrm{V}_{\text {urea }}$ than conventional (not-tunneled) two-lumen catheters. In the present study, double-lumen catheters were not used, each patient had two catheters - a right-sided thoracic and a femoral catheter of $25 \mathrm{~cm}$ length.

The other problem area of treatment-associated barriers relates to the function of the hemodialyzer. Severe bleeding diathesis is frequently present in critically ill patients. Consequently, for many patients heparin-free dialysis is prescribed for ARF. Heparin-free dialysis has been shown to cause a decreased patent fiber bundle volume despite no significant change in arterial or venous circuit pressure. In fact, there is concern that compromised dialyzer function during heparin-free dialysis affects treatment delivery in ARF patients treated with IHD [6]. Patients with risk for bleeding were excluded from the present study, all patients received systemic heparin. Finally, the calculated dialysis dose may not match the prescribed dose due to the fact that in vivo clearances are $10-15 \%$ lower than in vitro [23]. However, in this series the manufacturers of the dialyzers provided in vivo data from maintenance patients so that in vitro data did not need to be used. Patient hemodynamic instability may result in shorter treatments. Improved techniques of intermittent dialysis including variable sodium, calcium and ultrafiltration modeling combined with modifications of dialysate buffer or temperature allow safe therapy in the great majority of critically ill patients. In the present study, there was only a $10 \%$ incidence of significant 
hypotension in septic ARF patients. None of the sessions was prematurely terminated; blood flow rates were not changed during sessions with hypotensive episodes.

Practical difficulties to deliver dialysis dose in ARF patients may relate to intercompartmental urea disequilibrium, which can be conceptualized as delayed entrance of urea from relatively inaccessible body pools into the blood. This compartmentalization phenomenon may have several clinical implications. First, it is responsible for the well-described rebound in plasma urea concentration that occurs after dialysis, during which time the solute disequilibrium disappears. Most importantly, disequilibrium in urea distribution reduces the overall effective urea removal and overestimates delivered dialysis dose. Traditionally, many investigators have assumed that urea is distributed in the extracellular and intracellular spaces, with relative sequestration into the latter. However, Daugirdas and Schneditz [24] proposed that the regional blood flow differences can cause the aforementioned disequilibrium. In this model, urea disequilibrium is related to the degree of mismatch between blood supply and urea content in different body compartments. Specifically, although skeletal muscle is a large reservoir for both water and urea, in hypotensive, periph- erally vasoconstricted, critically ill patients, blood supply to this compartment is relatively low. This mismatch may be worsened in septic ICU patients who have further impairment of skeletal muscle perfusion due to vasopressor dependence. Currently, the effect of compartmentalization of urea on IHD dose delivery has not been fully investigated in the setting of critically ill patients with ARF, but it may not be as great as thought. Kanagasundaram et al. [10] determined average post-dialysis rebound (corrected for urea generation) to be only $11.4 \%$ which is similar to the values of $11.6 \%$ for maintenance hemodialysis outpatients using catheters. Moreover, measurements of the intercompartmental mass transfer coefficient, another measure of solute disequilibrium, provided only marginal support for urea disequilibrium.

In summary, this study provides evidence for the importance of patient-related characteristics such as the severity of acute underlying disease in affecting the delivered dose of IHD, as measured by spKt/ $\mathrm{V}_{\text {urea }}$. A number of factors may reduce the delivered dose to critically ill patients; no factor actually increases it, so the error will always be in the direction of a lower delivered dose when compared with the prescribed dose.

\section{References}

1 Weisbord SD, Palevsky PM: Acute renal failure in the intensive care unit. Semin Respir Crit Care Med 2006;27:262-273.

2 Paganini EP, Tapolyai M, Goormastic M, Halstenberg W, Kozlowski L, Leblanc M, Lee JC, Moreno L, Sakai K: Establishing a dialysis therapy/patient outcome link in intensive care unit acute dialysis for patients with acute renal failure. Am J Kidney Dis 1996; 28(suppl 3):S81-S89.

3 Schiffl H, Lang SM, Fischer R: Daily hemodialysis and the outcome of acute renal failure. N Engl J Med 2002;346:305-310.

4 Saudan P, Niederberger M, De Seigneux S, Romand J, Pugin J, Perneger T, Martin PY: Adding a dialysis dose to continuous hemofiltration increases survival in patients with acute renal failure. Kidney Int 2006; 70:13121317.

5 Leblanc M, Tapolyai M, Paganini EP: What dialysis dose should be provided in acute renal failure? A review. Adv Ren Replace Ther 1995;2:255-264.

6 Evanson JA, Himmelfarb J, Wingard R, Knights S, Shyr Y, Schulman G, Ikizler TA, Hakim RM: Prescribed versus delivered dialysis in acute renal failure patients. Am J Kidney Dis 1998;32:731-738.
7 Evanson JA, Ikizler TA, Wingard R, Knights S, Shyr Y, Schulman G, Himmelfarb J, Hakim RM: Measurement of the delivery of dialysis in acute renal failure. Kidney Int 1999; 55:1501-1508.

8 Jaber BL, King AJ, Cendoroglo M, CunniffJaber PJ, Balakrishnan VS, Ruthazer R, Pereira BJ: Correlates of urea kinetic modeling during hemodialysis in patients with acute renal failure. Blood Purif 2002;20:154160.

9 Liangos O, Rao M, Ruthazer R, Balakrishnan VS, Modi G, Pereira BJ, Jaber BL: Factors associated with urea reduction ratio in acute renal failure. Artif Organs 2004;28:10761081.

10 Kanagasundaram NS, Greene T, Larive AB, Daugirdas JT, Depner TA, Garcia M, Paganini EP: Prescribing an equilibrated intermittent hemodialysis dose in intensive care unit acute renal failure. Kidney Int 2003;64: 2298-2310.

11 Klouche K, Amigues L, Deleuze S, Beraud JJ, Canaud B: Complications, effects on dialysis dose, and survival of tunneled femoral dialysis catheters in acute renal failure. Am J Kidney Dis 2007;49:99-108.
12 American College of Chest Physicians/Society of Critical Care Medicine Consensus Conference: definitions for sepsis and organ failure and guidelines for the use of innovative therapies in sepsis. Crit Care Med 1992; 20:864-874.

13 Knaus WA, Wagner DP: Multiple systems organ failure: epidemiology and prognosis. Crit Care Clin 1989;5:221-232.

14 Watson PE, Watson ID, Batt RD: Total body water volumes for adult males and females estimated from simple anthropometric measurements. Am J Clin Nutr 1980;33:27-39.

15 Morbidity and mortality of renal dialysis: an NIH consensus conference statement. Consensus Development Conference Panel. Ann Intern Med 1994;121:62-70.

16 Renal Physician's Association Clinical Practice Guidelines Working Committee: Clinical Practice Guidelines on Adequacy of Hemodialysis. Clinical Practice Guideline; in Renal Physician's Association (ed): Clinical Practice Guideline No. 1. Dubuque, Kendall/ Hunt, 1996.

17 Daugirdas JT: Second generation logarithmic estimates of single-pool variable volume $\mathrm{Kt} / \mathrm{V}$ : an analysis of error. J Am Soc Nephrol 1993;4:1205-1213. 
18 Liang KY, Zeger SL: Longitudinal data analysis using generalized linear models. Biometrika 1986;73:13-22.

19 Marshall MR: Current status of dosing and quantification of acute renal replacement therapy. Part 1: mechanisms and consequences of therapy under-delivery. Nephrology 2006;11:171-180.

20 Himmelfarb J, Evanson J, Hakim RM, Freedman S, Shyr Y, Ikizler TA: Urea volume of distribution exceeds total body water in patients with acute renal failure. Kidney Int 2002;61:317-323
21 Clark WR, Mueller BA, Kraus MA, Macias WL: Dialysis prescription and kinetics in acute renal failure. Adv Ren Replace Ther 1997;4(suppl 1):64-71.

22 Ikizler TA, Sezer MT, Flakoll PJ, Hariachar S, Kanagasundaram NS, Gritter N, Knights S, Shyr Y, Paganini E, Hakim RM, Himmelfarb J: Urea space and total body water measurements by stable isotopes in patients with acute renal failure. Kidney Int 2004;65:725732 .
23 Paganini EP, Kanagasundaram NS, Larive B, Greene T: Prescription of adequate renal replacement in critically ill patients. Blood $\mathrm{Pu}$ rif 2001;19:238-244.

24 Daugirdas JT, Schneditz D: Overestimation of hemodialysis dose depends on dialysis efficiency by regional blood flow but not by conventional two pool urea kinetic analysis. ASAIO J 1995;41:M719-M724.

\section{Announcement}

The International Society for Hemodialysis (ISHD) will present the 2008 ISHD Belding Scribner Trailblazer Award to Dr. Jack Cimino on March 1, 2008 at the Annual Dialysis Conference (sponsored by the University of Missouri at Columbia, Mo. and to be held at the Rosen Shingle Creek Resort in Orlando, Fla., USA).

Dr. Cimino is honored for his exemplary, pioneer work on the development of an arteriovenous fistula for the purpose of dialysis access.

For more information please visit dialysis@health.missouri.edu.

Jointly sponsored by the Annual Dialysis Conference and the International Society for Hemodialysis, a 1-day symposium titled 'The Renaissance of Home Hemodialysis: Lessons from the World Over' will be held on March 1, 2008 at the Rosen Shingle Creek Resort, Orlando, Fla., USA.

Please visit dialysis@health.missouri.edu for more information.

The 17th International Vicenza Course on Hemodialysis and the 1st Congress of the International Society for Hemodialysis will be jointly sponsored by the Department of Nephrology, Dialysis and Transplantation, St. Bortolo Hospital, Vicenza, Italy, and the International Society for Hemodialysis.

The meeting will take place on June 3-6, 2008 at the Congress Center Ente Fiera, Vicenza, Italy.

All are welcome to attend. Information can be obtained from www.nefrologiavicenza.it or www.vicenzanephrocourses.com. 